\title{
Applying Stable Isotope Labeled Amino Acids in Micropatterned Hepatocyte Coculture to Directly Determine the Degradation Rate Constant for CYP3A4
}

\author{
Ryan H. Takahashi, Sheerin K. Shahidi-Latham, Susan Wong, and Jae H. Chang \\ Department of Drug Metabolism and Pharmacokinetics, Genentech Inc., South San Francisco, California
}

Received November 21, 2016; accepted March 10, 2017

\section{ABSTRACT}

The rate of enzyme degradation $\left(k_{\mathrm{deg}}\right)$ is an important input parameter for the prediction of clinical drug-drug interactions (DDIs) that result from mechanism-based inactivation or induction of cytochrome P450 (P450). Currently, a large range of reported estimates for CYP3A4 enzyme degradation exists, and consequently extensive uncertainty exists in steady-state predictions for DDIs. In the current investigations, the stable isotope labeled amino acids in culture technique was applied to a long-lived primary human hepatocyte culture, HepatoPac, to directly monitor the degradation of CYP3A4. This approach allowed selective isotope labeling of a population of de novo synthesized CYP3A4 and specific quantification of proteins with mass spectrometry to determine the CYP3A4 degradation within the hepatocytes. The $\boldsymbol{k}_{\text {deg }}$ estimate was $0.026 \pm 0.005$ hour $^{-1}$. This value was reproduced by cultures derived across four individual donors. For these cultures, the data indicated that CYP3A4 mRNA and total protein expression (i.e., labeled and unlabeled P450s), and activity were stable over the period where degradation had been determined. This $\boldsymbol{k}_{\text {deg }}$ value for CYP3A4 was in good agreement with recently reported values that used alternate analytical approaches but also employed micropatterned primary human hepatocytes as the in vitro model.

\section{Introduction}

Understanding the mechanism of inhibition is important when assessing the magnitude of cytochrome P450 (P450)-mediated drugdrug interaction (DDI). For example, it has been shown that the extent of DDIs is underpredicted when a quasi-irreversible inhibitor such as troleandomycin or an irreversible inhibitor such as mibefradil are assumed to block CYP3A4 via a reversible process (Ito et al., 2004; Fahmi et al., 2008). Quantitative assessment of irreversible inhibition takes into account the concentration of the perpetrator and kinetic parameters that characterize the in vitro potency of the perpetrator on the enzyme (Kitz and Wilson, 1962; Silverman, 1995). In addition, because the perpetrator inactivates the protein to affect its steady-state concentration, the $\mathrm{P} 450$ degradation rate constant $\left(k_{\mathrm{deg}}\right)$ is another parameter that requires consideration (Mayhew et al., 2000). It is typically assumed that $k_{\mathrm{deg}}$ is a constant value; however, sensitivity analyses have demonstrated that differing values of $k_{\text {deg }}$ can have a profound effect on the extent of DDI predictions (Galetin et al., 2006; Wang, 2010). Thus, ambiguity in which $k_{\mathrm{deg}}$ value is most appropriate for the quantitative assessment of irreversible inhibition remains a confounding factor for high confidence in the prospective prediction of DDIs.

A wide range of $k_{\mathrm{deg}}$ values have been reported for CYP3A4 (Yang et al., 2008). Because in vivo measurement in humans is challenging, several in vitro matrices have been used to determine the $k_{\mathrm{deg}}$ values.

https://doi.org/10.1124/dmd.116.074393

SThis article has supplemental material available at dmd.aspetjournals.org.
Although hepatocytes may be the best characterized model for the function and dynamics of the liver, the observed declines in P450 protein expression and activities in regular short-lived cultures have limited their suitability for determining the true half-life $\left(t_{1 / 2}\right)$ of P450 turnover (Correia, 1991). The development and adoption of long-lived hepatocyte models have been prompted by their superiority in determining metabolic stabilities and generating metabolites for low intrinsic clearance compounds (Chan et al., 2013; Ballard et al., 2016). Therefore, a long-lived hepatocyte model such as the HepatoPac, which is a specialized coculture system that sustains P450 activities for several days (Khetani and Bhatia, 2008), may be a more suitable model for characterizing the dynamics of $\mathrm{P} 450$ turnover.

The stable isotope labeled amino acids in culture (SILAC) technique with HepatoPac was applied to determine the $k_{\text {deg }}$ value. SILAC utilizes the cellular machinery for de novo protein synthesis to take up isotopically labeled essential amino acids from media and incorporate them into the proteome; thus, creating a population of proteins from a determined culture period to be identified. Mass spectrometry is then used to individually monitor the labeled and unlabeled proteins based on mass shifts, and the dynamics of protein expression can be quantified. SILAC was first demonstrated to describe protein upregulation during muscle differentiation (Ong et al., 2002) and was subsequently applied to investigate protein turnover in yeast (Pratt et al., 2002) and human adenocarcinoma cells (Doherty et al., 2009). CYP3A4 is responsible for metabolizing the largest proportion of drugs, and therefore has been the focus for many DDI studies. In the current work, the CYP3A4 $k_{\text {deg }}$ value was determined by measuring protein turnover.

ABBREVIATIONS: ACN, acetonitrile; DDI, drug-drug interaction; $6 \mathrm{HT}, 6 \beta$-hydroxytestosterone; $k_{\text {deg, }}$, degradation rate constant; LC-MS/MS, liquid chromatography-tandem mass spectrometry; P450, cytochrome P450; SIL, stable isotope label; SILAC, stable isotope labeled amino acids in culture; SRM, selected reaction monitoring; $t_{1 / 2}$, half-life. 


\section{Material and Methods}

Materials Used. ${ }^{13} \mathrm{C}_{6}$ Arginine and ${ }^{13} \mathrm{C}_{6}$ lysine were purchased from Cambridge Isotope Laboratories (Tewksbury, Massachusetts). Testosterone, $6 \beta$-hydroxytestosterone (6HT), and $\mathrm{d}_{7}-6 \mathrm{HT}$ were purchased from Sigma-Altrich (St. Louis, MO), Cerilliant (Round Rock, TX), and Corning/Gentest (Woburn, $\mathrm{OH})$, respectively. The stable isotope labeled peptides $\left[\operatorname{Arg}\left(\mathrm{U}^{13} \mathrm{C}_{6},{ }^{15} \mathrm{~N}_{4}\right)\right]$ used as internal standards were custom synthesized with purities $>95 \%$ by CPC Scientific Inc. (Sunnyvale, CA) or New England Peptide (Gardner, MA). All other chemicals were purchased from vendors at the reagent grade or better.

Cell Cultures. Human HepatoPac cultures were prepared and maintained by Hepregen (Medford, MA). Four individual donors of hepatocytes (3121A, 3121B, 4202, and 4297) (donor information provided in Supplemental Table 1) were tested with 32,000 hepatocytes seeded to each well of a 24-well plate. In preliminary experiments, cell samples at days 3 and 7 following setup of the cultures were collected. Increasing incorporation of heavy amino acids was observed between days 3 and 7 and to a much lesser extent between days 7 and 10, indicating that steady state had been reached by day 7 (data not shown). However, to ensure steady-state conditions, the cocultures were maintained with the stable isotope label for 10 days before initiating the experiment by replacing them with a nonstable isotope label. For seeding and maintenance of the cultures until day 10 of the culture, proprietary seeding and maintenance media were prepared by replacing the lysine and arginine with their stable isotope ${ }^{13} \mathrm{C}_{6}$ labeled equivalents. Media changes were conducted every other day. On day 10 of the culture, the stable isotope label (SIL)-containing media was replaced with the non-SILcontaining regular maintenance media and changed every other day. At the termination of a culture, CYP3A4 activities were determined followed by collection of HepatoPac lysates. In triplicate, four wells were pooled in a single volume for each donor at each time point. The medium was removed from the culture and the cells in each well were washed with phosphate-buffered saline. Collection buffer $(200 \mu \mathrm{l} ; 1 \mathrm{mM}$ EDTA, $0.1 \mathrm{mM}$ dithiothreitol, $2 \mu \mathrm{g} / \mathrm{ml}$ leupeptin, $250 \mathrm{mM}$ sucrose, and $150 \mathrm{mM} \mathrm{KCl}, \mathrm{pH}$ 7.4) was added to one cell and the well contents were scraped. The cell suspension was transferred to the next well and collection was repeated until four wells were pooled. The wells were washed with an additional $200 \mu \mathrm{l}$ volume of buffer to collect residual cells, and the cell suspension and wash were pooled and stored at $-80 \mathrm{C}$ until analysis.

CYP3A4 Activity Measurement. CYP3A4 activity was measured by monitoring the formation of $6 \mathrm{HT}$ from each cell culture immediately prior to cell collection and lysis as described previously (Halladay et al., 2012). Testosterone solution was prepared in serum-free media at a final concentration of $200 \mu \mathrm{M}$. The medium was removed from the wells and $300 \mu \mathrm{l}$ of testosterone solution was added to the hepatocytes. The plates were incubated for 1 hour at $37^{\circ} \mathrm{C}$ with $10 \% \mathrm{CO}_{2}$. The supernatants were collected and stored at $-80^{\circ} \mathrm{C}$ until analysis. For analysis, the HepatoPac testosterone supernatants were diluted $50 \times$ with water:acetonitrile ( $\mathrm{ACN}$ ) (3:1 by volume) that contained $1 \mu \mathrm{M} \mathrm{d}_{7}-6 \mathrm{HT}$ as an internal standard. The formation of $6 \mathrm{HT}$ was measured by liquid chromatography-tandem mass spectrometry (LC-MS/MS) by selected reaction monitoring (SRM) in positive ion electrospray mode [305.5 $\rightarrow 269.1$ and $312.5 \rightarrow$ 276.1 ( $\mathrm{d}_{7}$ internal standard)] using a 5500 QTRAP (AB Sciex, Foster City, CA) attached to a CTC PAL Autosampler (LEAP Technologies, Carrboro, NC) with a 1290 pump (Agilent, Santa Clara, CA). Samples were injected to a Kinetex XB-C18 $1.7 \mu \mathrm{m}, 50 \times 2.1 \mathrm{~mm}$, column (Phenomenex, Torrance, CA) and chromatographed with a gradient elution of water and ACN containing $0.1 \%$ formic acid. The flow rate was $0.75 \mathrm{ml} / \mathrm{min}$ over a total run time of 3.5 minutes. Concentrations were calculated by comparing the samples against a calibration curve of $6 \mathrm{HT}$ constructed by weighted $\left(1 / x^{2}\right)$ linear regression of analyte-tointernal standard peak area ratio versus nominal concentrations $(0.1-100 \mu \mathrm{M})$. In vitro formation rates were calculated from the concentration of metabolite formed in 60 minutes by 32,000 hepatocytes and scaled to one million hepatocytes.

LC-MS/MS Measurement of P450 Proteins. Pellets of the HepatoPac lysates $(125 \mu \mathrm{l})$ were prepared by centrifuging aliquots of the samples at $9000 \mathrm{~g}$ for 20 minutes at $4^{\circ} \mathrm{C}$. The supernatants were removed, which showed no detectable $\mathrm{P} 450$ protein in the pilot experiments. The trypsin digestion procedure employed is similar to that reported by other investigators for the absolute quantification of hepatic P450s (Wang et al., 2008; Michaels and Wang, 2014). The sample pellets were resuspended in ammonium bicarbonate buffer $(50 \mathrm{mM})$ and proteins (target $40 \mu \mathrm{g}$ ) were denatured by adding dithiothreitol $(13.3 \mathrm{mM}$ final) and heating to $60^{\circ} \mathrm{C}$ for 60 minutes. Samples were alkylated with the addition of iodoacetamide (19.3 $\mathrm{mM}$ final), left in the dark at room temperature for 30 minute, and then digested with trypsin (400 ng, 1:100 trypsin: protein) overnight at $37^{\circ} \mathrm{C}$. An internal standard solution of ACN containing $0.2 \mathrm{fmol}$ of ${ }^{13} \mathrm{C}_{6}^{15} \mathrm{~N}$-labeled peptide (EVTNFLR) was added to the samples and then the samples were centrifuged and the supernatants were removed for analysis. Samples were analyzed by LC-MS/MS using the setup as described for monitoring 6HT. Samples were injected to a Kinetex XB-C18 $2.6 \mu \mathrm{m}$, $100 \times 2.1 \mathrm{~mm}$, column (Phenomenex). The solvent gradient started at 95\% A (water containing $0.1 \%$ formic acid) $/ 5 \% \mathrm{~B}$ ( $\mathrm{ACN}$ containing $0.1 \%$ formic acid), which was held for 2 minutes, and was then increased to $20 \% \mathrm{~B}$ at 25 minutes and $50 \%$ B at 28 minutes before the column was flushed with $98 \%$ $\mathrm{B}$ and re-equilibrated at $5 \% \mathrm{~B}$. The flow rate was $0.65 \mathrm{ml} / \mathrm{min}$ over a total run time of 35 minutes. Four unique peptides (amino acids 131-141, 244-250, 380-390, and 407-413) were selected to monitor CYP3A4 based on their selectivity, stability, and reproducible release with trypsin digestion. The identity of the peptides and their SRM parameters used for LC-MS/MS protein measurements are provided in Supplemental Table 2. SRM data were imported into Skyline (MacCoss Lab Software, Seattle, WA) for processing. The most intense SRM was used for quantitation and two other SRMs were used as qualifier transitions (Supplemental Fig. 1). To calculate P450 expression, the ratio of the unlabeled or SIL-containing peptide to the internal standard peptide was multiplied by $0.2 \mathrm{fmol}$ (single point calibration) and scaled to one million hepatocytes.

mRNA Determination. Aliquots of the HepatoPac lysates were used for determination of P450 mRNA levels. The cell lysates were combined with $100 \mu \mathrm{l}$ of Procarta cell lysis buffer. A QuantiGene Plex 2.0 Assay Kit based on the bead technology from Affymetrix (Santa Clara, CA) was used in a similar manner as previously described (Halladay et al., 2012). The mRNA measurements for CYP3A4 were normalized to those for glyceraldehyde-3-phosphate dehydrogenase, and the normalized expression was reported relative to day 10 for the same hepatocyte donor.

Data Analysis and Half-Life Calculations. For each sample at a specific time point, the peak areas for corresponding unlabeled and SIL-containing peptides were summed. The peak area for the SIL-containing peptide, which corresponded to the pre-existing P450, was then calculated as a percentage of the total P450. The resulting percentage of existing peptide/protein remaining was transformed by taking the natural logarithm plotted against time. The half-life values were derived $\left[t_{1 / 2}=-\ln (2) / k\right]$ from the slope of the line $(k)$ determined by linear regression using Prism 6 (GraphPad, San Diego, CA). When calculating the slope, each measurement was normalized by the total protein level and degradation was calculated from the percentage of depletion over time as required for this first-order kinetic process. Therefore, determinations

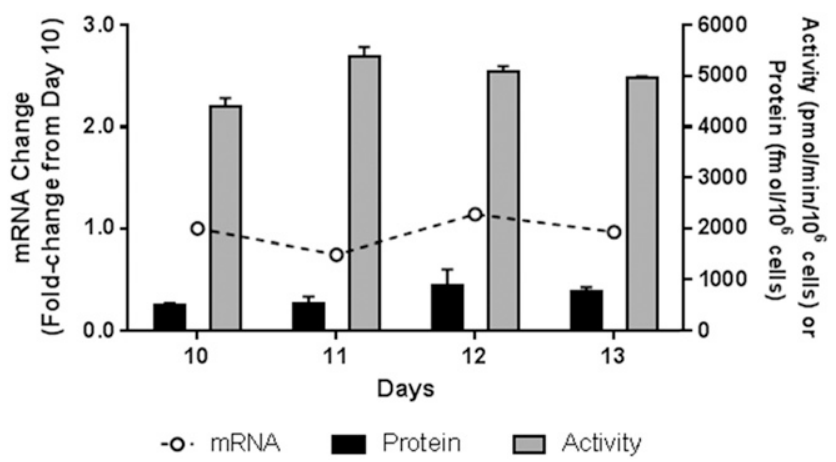

Fig. 1. Representative CYP3A4 stability data for human HepatoPac cocultures through the time period used for $\mathrm{P} 450$ degradation rate determinations. Data are mean from $n=3$ replicates with error bars indicating S.D. mRNA expressions are normalized to day 10 (left axis) and CYP3A4 activity (measured as testosterone-6 $\beta$ hydroxylation) and protein expression (right axis) are normalized to one million hepatocytes. Protein expressions were determined by mass spectrometry-determined abundance of peptide EVTNFLR relative to stable isotope $\left({ }^{13} \mathrm{C}_{6}^{15} \mathrm{~N}\right)$ labeled peptide used as an internal standard calibrator. Data shown are for one hepatocyte donor (3121A) 

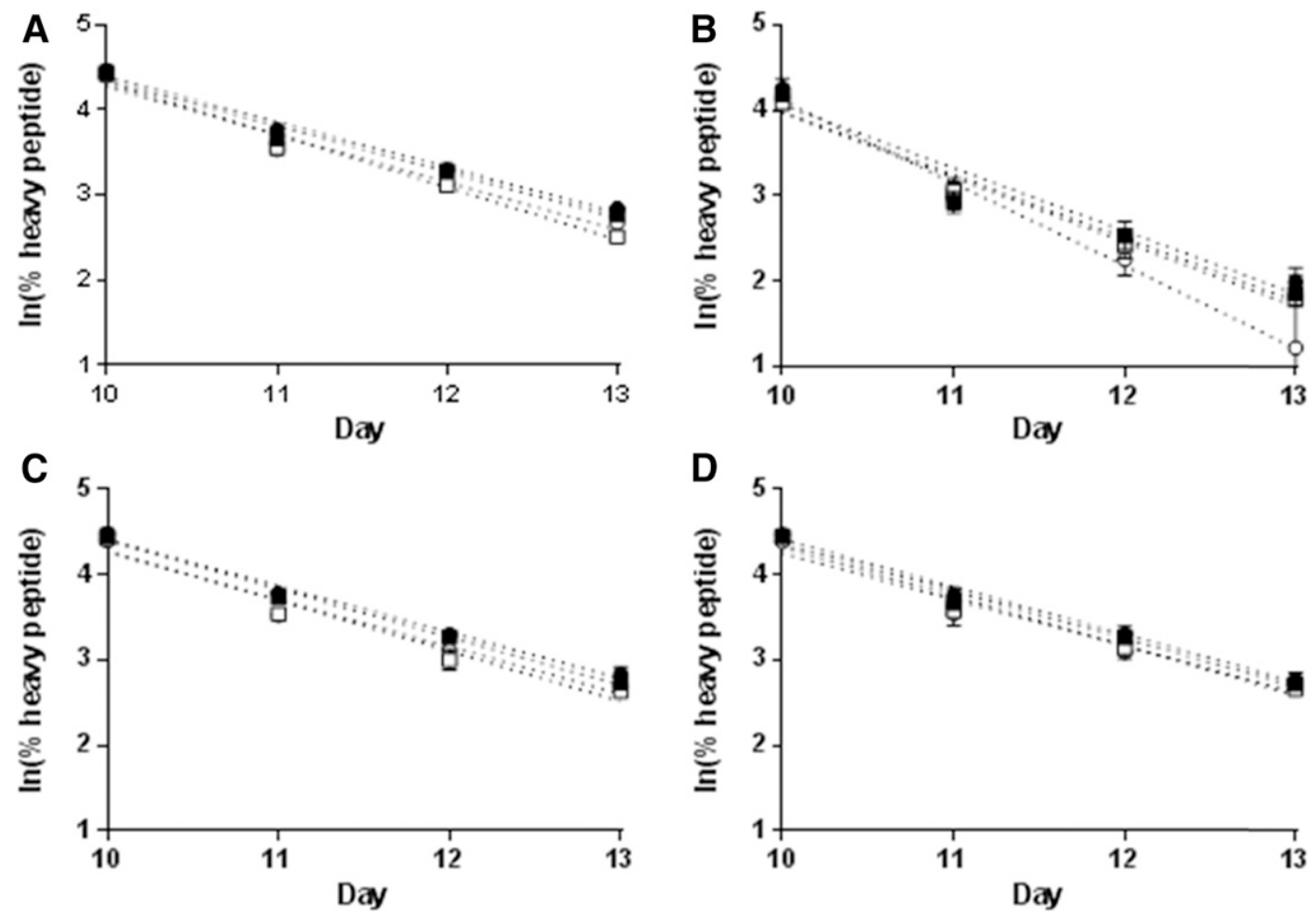

Fig. 2. P450 degradation of CYP3A4 as measured by SIL-containing peptide depletion for four donors with protein-specific peptides: (A) SLLSPTFTSGK, (B) EVTNFLR, (C) VEINGMFIPK, and (D) YWTEPEK. Labeled peptide abundances are expressed as percentages of the total measured peptide, $n=3$ per time point with error bars representing S.D. Days are marked from the start of the HepatoPac cultures with SIL amino acids supplemented for days 0-10. Each line represents the linear regression for an individual donor from which the slope was used to calculate the protein $t_{1 / 2}$.

of $k_{\mathrm{deg}}$ and $t_{1 / 2}$ were independent of the absolute expression levels since the percentages of population protein (\% heavy peptide) were determined and used to calculate the rates of protein turnover.

\section{Results and Discussion}

Stability Assessment of HepatoPac Cultures. There have been multiple publications that have demonstrated P450 levels in HepatoPac cultures reach steady state by day 10 . For example, HepatoPac cocultures have been extensively characterized to show that there is sustained and stable expression and activities of P450s by day 7 (product information sheet). In addition, in their determination of $k_{\mathrm{deg}}$, Ramsden et al. (2015) and Dixit et al. (2016) maintained the HepatoPac cultures for 9 days before starting their treatment. Ramsden et al. (2015) provided more detail to confirm that P450 was at steady state by showing that there were no significant differences in CYP3A4 activity from day 7 to 28 (i.e., $<20 \%$ ), but that the biggest differences (i.e., $>40 \%$ ) were observed only between days 7 and 8 and after day 20. In addition to these reports, preliminary experiments from this laboratory demonstrated stable cultures in SILAC conditions with visual inspection of cell morphology and measurement of cell viability by ATP presence (inhouse data). In the current experiments, mRNA, protein, and activity of CYP3A4 were monitored to confirm the stability of the hepatocyte culture over the experimental time frame (Fig. 1; Supplemental Table 3). The mean CYP3A4 content in HepatoPac was estimated to be 401-703 pmol per million hepatocytes, assuming 32,000 hepatocytes per HepatoPac well. The observed rates for $6 \mathrm{HT}$ formation among the four donors were $3.5-4.8 \mathrm{nmol} / \mathrm{min}^{-1}$ per million hepatocytes. In general, cultures with higher CYP3A4 content had correspondingly higher rates for $6 \mathrm{HT}$ formation. It is worth mentioning that since $\beta$-glucuronidase and sulfatase were not added to the incubation, the amount of total $6 \mathrm{HT}$ may be underestimated. However, the stability of CYP3A4 mRNA and protein give some confidence that $6 \mathrm{HT}$ is likely reflecting CYP3A4 activity. These readouts demonstrated that the cultures had achieved a stable expression level and function of P450s by day 10. The mRNA and protein expressions were not measured directly on the cultures. Instead, they were measured following cell lysate collection, pooling, and processing; therefore, intersample variability was expectedly higher. However, this variability would not bias the determination of degradation rates since the measured stable isotope-containing peptides were normalized to the total measured peptide for each sample.

Incorporation of SIL to CYP3A4. On day 10 of the HepatoPac culture (initial day of sample collection and protein measurement), the

TABLE 1

Degradation rates $\left(k_{\mathrm{deg}}\right)$ and half-lives $\left(t_{1 / 2}\right)$ determined using HepatoPac-SILAC for CYP3A4

Data were calculated from four donors and are reported as mean and S.D. The average $k_{\text {deg }}$ and $t_{1 / 2}$ values determined for the three nonarginine-containing CYP3A4 peptides were $0.023 \pm$ $0.002 \mathrm{~h}^{-1}$ and $29.7 \pm 2.3$ hours, respectively. The $k_{\operatorname{deg}}$ and $t_{1 / 2}$ values for the arginine-containing peptide (EVTNFLR) were $0.033 \pm 0.005 \mathrm{~h}^{-1}$ and $21.0 \pm 2.6$ hours, respectively. Recent $k_{\mathrm{deg}}$ and $t_{1 / 2}$ value determinations for CYP3A4 using HepatoPac by mRNA following siRNA or interleukin 6 inhibition were $0.0240 \mathrm{~h}^{-1}$ and 28.9 hours, respectively (Ramsden et al., 2015); and by mRNA following induction they were $0.0261 \mathrm{~h}^{-1}$ and 26 hours, and 49 hours by activity following rifampicin induction (Dixit et al., 2016).

\begin{tabular}{clcc}
\hline P450 & \multicolumn{1}{c}{ Peptide $^{a}$} & $k_{\mathrm{deg}}$ & $t_{1 / 2}$ \\
\hline & & $h^{-1}$ & $h$ \\
CYP3A4 & SLLSPTFTSGK & $0.023 \pm 0.002$ & $29.6 \pm 1.9$ \\
CYP3A4 & DVEINGMFIPK & $0.023 \pm 0.001$ & $29.6 \pm 1.0$ \\
CYP3A4 & YWTEPEK & $0.023 \pm 0.001$ & $29.8 \pm 0.8$ \\
\hline
\end{tabular}

${ }^{a}$ The SRM parameters of the specific peptides used for LC-MS/MS protein measurements are provided in Supplemental Table 2. 
mean incorporation of ${ }^{13} \mathrm{C}_{6}$-lysine peptides was $84.8 \%$, and for the ${ }^{13} \mathrm{C}_{6^{-}}$ arginine peptide it was $66.2 \%$. On day 13 of the culture (final day of sample collection and measurement), the incorporation of ${ }^{13} \mathrm{C}_{6}$-lysine peptides was $15.2 \%$, and for the ${ }^{13} \mathrm{C}_{6}$-arginine peptide it was $6.0 \%$. There were no trends observed that indicated clear differences among donors. Variability among donors in incorporation of SIL (calculated as CV) were less than $5 \%$ on day 10 and less than $10 \%$ on day 13 .

Determination of CYP3A4 Degradation Rate. The plots for rates of decline in the SIL-containing peptides for the four HepatoPac donors are presented in Fig. 2 and a summary of the data is provided in Supplemental Table 4. Between the four hepatocyte donors, the variability was minimal and there was good consistency between the three ${ }^{13} \mathrm{C}_{6}$-lysine-containing peptides. Given the apparent lower and more variable incorporation of ${ }^{13} \mathrm{C}_{6}$-arginine, it was not included in the calculations of $t_{1 / 2}$ and degradation rates. The apparent $t_{1 / 2}$ values of CYP3A4 protein in HepatoPac were $29.7 \pm 2.3$, and corresponding estimates for the $k_{\mathrm{deg}}$ values were $0.023 \pm 0.002$ (Table 1).

Degradation rates for CYP3A4 were recently reported by two other research groups (Ramsden et al., 2015; Dixit et al., 2016). Coincidentally, these investigators also used HepatoPac. This reflects the recent development and adoption of long-lived primary human hepatocyte cultures. The protein dynamics were directly measured in cultures in the current study, whereas the other investigators determined the $k_{\text {deg }}$ values by mRNA and/or activity changes utilizing HepatoPac during the loss and successive recovery of $\mathrm{P} 450$, following treatment with suppressors or inducers. Despite these differences, there is remarkable agreement in determinations with the $t_{1 / 2}$ values of CYP3A4 ranging from 22 to 49 hours. The convergence of values for $k_{\text {deg }}$ CYP3A4 from various approaches and multiple laboratories provides added confidence in these determinations, especially in light of the 3-fold range of previously reported estimates for CYP3A4 enzyme degradation (Wong, 2011). The limits for the translatability of this $k_{\mathrm{deg}}$ value for steady-state predictions of DDI will need to be determined since it currently reflects a single in vitro model and a limited number of hepatocyte donors. However, the current and apparently consensus $k_{\mathrm{deg}}$ values should allow future work to focus on systematically evaluating the sensitivity of the predictive models for DDIs to other parameters. Toward advancing the understanding of the relevance of the in vitro $k_{\text {deg }}$ values in HepatoPac, our laboratory is currently applying SILAC to investigate if there is any potential impact of perpetrators such as irreversible inhibitors and inducers on the CYP3A4 $k_{\mathrm{deg}}$ value in this model. In addition, in vivo SILAC experiments are being conducted in animal models expressing human orthologs of $\mathrm{P} 450$ proteins to determine the $k_{\mathrm{deg}}$ values.

The presented methodology has wide applicability to determining degradation rates for many proteins of interest and is amenable to multiplexing to measure several target proteins in each study. The current results reveal HepatoPac to be a suitable stable cell system that can be sustained over several protein half-lives. Adequate incorporation of the SIL into the proteome depends on the rates of protein synthesis, degradation, and turnover. Within a 10-day period of HepatoPac culture stabilization, high incorporation $(\geq 85 \%)$ of peptides for lysine incorporation was achieved. This incorporation and the subsequent degradation were largely reproducible between hepatocyte donors, which confirmed that the machinery for protein turnover had been well-maintained throughout hepatocyte isolation, preservation, and culture. This also suggested that HepatoPac may be a suitable model to investigate modulation of de novo protein synthesis and degradation by drugs or other treatment, and we are currently undertaking such investigations. An observation that should be considered in subsequent experiments is lower and more variable incorporation of arginine compared with lysine. This likely is due to an alternate source of arginine within the hepatocyte such that cells were not restricted to exclusive use of exogenously supplemented arginine, suggesting the limited utility of the arginine label.

In the case of CYP3A4 degradation, comparable estimates for $k_{\mathrm{deg}}$ between the current and previous methods indicate that mRNA and enzymatic activity may be appropriate surrogate measurements for protein dynamics. However, there may be limitations on the universal application of the previous methods to study protein kinetics. In particular, because the recovery of CYP3A4 expression was monitored following varying the in vitro conditions via inhibition or induction, these approaches do not differentiate between pre-existing and newly formed proteins. Therefore, measurement of $k_{\text {deg }}$ can be inaccurate if the perpetuating compound affects the protein synthesis rate. Moreover, the previous methods relied on well-characterized selective substrates, inducers, and/or inhibitors of CYP3A4. For proteins that have been rarely studied, these tools may not yet be identified. In these cases, direct protein measurement, as applied in the current study, may be a preferable strategy. Furthermore, the ability to multiplex the peptide analysis, especially if a nontargeted proteomics approach is taken, presents an opportunity to simultaneously obtain a comprehensive profile of cellular protein dynamics.

In this study, the utility of long-lived hepatocyte cocultures to directly measure the turnover of P450s by applying SILAC and mass spectrometry was demonstrated. In concept, this approach is immediately applicable to other drug metabolizing enzymes and transporter proteins that maintain expression and functionality in these in vitro models. The estimate of 29.7 hours for the $t_{1 / 2}$ value of CYP3A4 agrees remarkably well with recently reported values by other investigators who applied HepatoPac technology to monitor protein dynamics by alternate approaches.

\section{Acknowledgments}

We thank Dr. Donald Kirkpatrick for valuable scientific input.

\section{Authorship Contributions:}

Participated in research design: Takahashi, Shahidi-Latham, Wong, Chang. Conducted experiments: Takahashi, Wong.

Performed data analysis: Takahashi, Wong.

Wrote/contributed to the writing of the manuscript: Takahashi, ShahidiLatham, Wong, Chang.

\section{References}

Ballard TE, Wang S, Cox LM, Moen MA, Krzyzewski S, Ukairo O, and Obach RS (2016) Application of a micropatterned cocultured hepatocyte system to predict preclinical and humanspecific drug metabolism. Drug Metab Dispos 44:172-179.

Chan TS, Yu H, Moore A, Khetani SR, and Tweedie D (2013) Meeting the challenge of predicting hepatic clearance of compounds slowly metabolized by cytochrome P450 using a novel hepatocyte model, HepatoPac. Drug Metab Dispos 41:2024-2032.

Correia MA (1991) Cytochrome P450 turnover. Methods Enzymol 206:315-325.

Dixit V, Moore A, Tsao H, and Hariparsad N (2016) Application of micropatterned cocultured hepatocytes to evaluate the inductive potential and degradation rate of major xenobiotic metabolizing enzymes. Drug Metab Dispos 44:250-261.

Doherty MK, Hammond DE, Clague MJ, Gaskell SJ, and Beynon RJ (2009) Turnover of the human proteome: determination of protein intracellular stability by dynamic SILAC. J Proteome Res 8:104-112.

Fahmi OA, Maurer TS, Kish M, Cardenas E, Boldt S, and Nettleton D (2008) A combined model for predicting CYP3A4 clinical net drug-drug interaction based on CYP3A4 inhibition, inactivation, and induction determined in vitro. Drug Metab Dispos 36:1698-1708.

Galetin A, Burt H, Gibbons L, and Houston JB (2006) Prediction of time-dependent CYP3A4 drug-drug interactions: impact of enzyme degradation, parallel elimination pathways, and intestinal inhibition. Drug Metab Dispos 34:166-175.

Halladay JS, Wong S, Khojasteh SC, and Grepper S (2012) An 'all-inclusive' 96-well cytochrome P450 induction method: measuring enzyme activity, mRNA levels, protein levels, and cytotoxicity from one well using cryopreserved human hepatocytes. J Pharmacol Toxicol Methods 66:270-275.

Ito K, Brown HS, and Houston JB (2004) Database analyses for the prediction of in vivo drug-drug interactions from in vitro data. Br J Clin Pharmacol 57:473-486.

Khetani SR and Bhatia SN (2008) Microscale culture of human liver cells for drug development. Nat Biotechnol 26:120-126.

Kitz R and Wilson IB (1962) Esters of methanesulfonic acid as irreversible inhibitors of acetylcholinesterase. J Biol Chem 237:3245-3249. 
Mayhew BS, Jones DR, and Hall SD (2000) An in vitro model for predicting in vivo inhibition of cytochrome P450 3A4 by metabolic intermediate complex formation. Drug Metab Dispos 28 : $1031-1037$.

Michaels S and Wang MZ (2014) The revised human liver cytochrome P450 "Pie": absolute protein quantification of CYP4F and CYP3A enzymes using targeted quantitative proteomics. Drug Metab Dispos 42:1241-1251.

Ong SE, Blagoev B, Kratchmarova I, Kristensen DB, Steen H, Pandey A, and Mann M (2002) Stable isotope labeling by amino acids in cell culture, SILAC, as a simple and accurate approach to expression proteomics. Mol Cell Proteomics 1:376-386.

Pratt JM, Petty J, Riba-Garcia I, Robertson DH, Gaskell SJ, Oliver SG, and Beynon RJ (2002) Dynamics of protein turnover, a missing dimension in proteomics. Mol Cell Proteomics 1 579-591.

Ramsden D, Zhou J, and Tweedie DJ (2015) Determination of a degradation constant for CYP3A4 by direct suppression of mRNA in a novel human hepatocyte model, HepatoPac. Drug Metab Dispos 43:1307-1315.

Silverman RB (1995) Mechanism-based enzyme inactivators. Methods Enzymol 249:240-283.

Wang MZ, Wu JQ, Dennison JB, Bridges AS, Hall SD, Kornbluth S, Tidwell RR, Smith PC, Voyksner RD, Paine MF, et al. (2008) A gel-free MS-based quantitative proteomic approach accurately measures cytochrome $\mathrm{P} 450$ protein concentrations in human liver microsomes. Proteomics 8:4186-4196.

Wang YH (2010) Confidence assessment of the Simcyp time-based approach and a static mathematical model in predicting clinical drug-drug interactions for mechanism-based CYP3A inhibitors. Drug Metab Dispos 38:1094-1104.

Wong SG (2011) Prediction of drug-drug interactions arising from mechanism-based inactivation key input parameters and impact on risk assessment. Curr Drug Metab 12:871-890.

Yang J, Liao M, Shou M, Jamei M, Yeo KR, Tucker GT, and Rostami-Hodjegan A (2008) Cytochrome P450 turnover: regulation of synthesis and degradation, methods for determining rates, and implications for the prediction of drug interactions. Curr Drug Metab 9 384-394.

Address correspondence to: Dr. Jae H. Chang, Drug Metabolism and Pharmacokinetics, Genentech, Inc., 1 DNA Way, South San Francisco, CA 94080. E-mail: jaechang@gmail.com 